\title{
New Feature Set for Better Representation of Dynamic of RR Intervals in Poincare Plot
}

\author{
Sadaf Moharreri ${ }^{1}$, Nader Jafarnia Dabanloo ${ }^{2}$, Shahab Rezaei ${ }^{3}$, Saman Parvaneh ${ }^{2,4}$ \\ ${ }^{1}$ Islamic Azad University, Khomeini Shahr Branch, Isfahan, Iran \\ ${ }^{2}$ Islamic Azad University, Science and Research Branch, Tehran, Iran \\ ${ }^{3}$ Islamic Azad University, Central Tehran Branch, Tehran, Iran \\ ${ }^{4}$ Philips Research North America, Cambridge, MA, USA
}

\begin{abstract}
Traditional features extracted from Poincare plot (e.g., SD1 and SD2) ignore temporal information and only quantify point distribution. In this article, a new feature set is proposed to capture the dynamic of point distribution in the Poincare plot. For quantification of temporal information in Poincare plot, extracted ADP features (angle, direction, and position) from point distribution in the Poincare plot were used with K-Nearest Neighbor (KNN) classifier for classification of four cardiac condition obtained from Physionet database (normal sinus rhythm, myocardial infarction, congestive heart failure, and atrial fibrillation). KNN was trained on $70 \%$ of data as a train set, and the accuracy was evaluated on $30 \%$ of data as a test set. Accuracy of classification were $94.8 \%$ and $95.58 \%$ for training and test set, respectively. Furthermore, ADP features were used for creating a new map that represents the temporal information of points in Poincare plot.
\end{abstract}

\section{Introduction}

Heart Rate Variability (HRV) is the variation in the time series of RR intervals [1] and is an indicator of heart's condition [2]. It has been proved that nonlinear analysis of HRV provides more valuable information for the physiological interpretation of heart rate fluctuations compared to linear HRV measures [3]. One of the useful nonlinear analysis of HRV is Poincare plot, plot of each RR interval against next RR interval [4]. Woo et al. were the first group who used Poincare plot for evaluating the differences between healthy subjects and heart failure patients [5]. Poincare plot is a geometrical representation of RR time series to demonstrate patterns of heart rate dynamics resulting from nonlinear processes [6]. Tulppo et al. [7] fitted an ellipse to the point distribution in the
Poincare plot and defined two standard descriptors (SD1 and SD2) for quantification of the Poincare plot geometry. These standard descriptors represent the minor axis and the major axis of the ellipse and guide the visual inspection of the distribution. Brennan et al. proved that although Poincare plot is a nonlinear representation of RR intervals, but SD1 and SD2 cannot describe these nonlinear behavior and are linear and statistic parameters [8]. Furthermore, these descriptors ignore temporal information and only quantify point distribution [9].

In addition to geometric features that was proposed by our team previously [10-13], a new feature set is proposed in this article to capture the temporal information in a Poincare plot by considering the angle between consecutive points, the direction of the trajectory, and the position of points in relation to the line of identity. These features were used to create ADP Map (angle, direction, and position map) for a new representation of RR points.

Performance of proposed feature set was evaluated in distinguishing four cardiac condition including normal sinus rhythm (NSR), acute myocardial infarction (MI), congestive heart failure (CHF), and atrial fibrillation (AF).

\section{Method and Data}

\subsection{Method}

For extraction of the new feature set and creating new map, quantification of temporal information/dynamics of the points in Poincare plot were the focus of this article. For capturing dynamic information, three features were calculated between every three consecutive points in Poincare plot: angle, direction of the trajectory, and the location of middle point in relation to the line of identity. For visualization purpose, these features were used to create a 3D map (ADP map). 


\subsubsection{Angle}

For defining the angle between every three consecutive points, points in Poincare plot were connected in their temporal orders as is shown in Figure 1 and the angle between two sequential vectors was measured.

For measuring the angle between two vectors, first length of two vectors which connects the two sequential points should be measured. This can be obtained by measuring the distance between two points as:

$$
D_{i}=\sqrt{\left(R R_{i}-R R_{i+1}\right)^{2}+\left(R R_{i+1}-R R_{i+2}\right)^{2}}
$$

and then the angle between two consecutive vectors is defined as:

$$
\alpha_{i}=\cos ^{-1}\left(\frac{D_{i}^{2}+D_{i+1}^{2}-\left(\left(R R_{i}-R R_{i+2}\right)^{2}+\left(R R_{i+1}-R R_{i+3}\right)^{2}\right)}{2 D_{i} D_{i+1}}\right)
$$

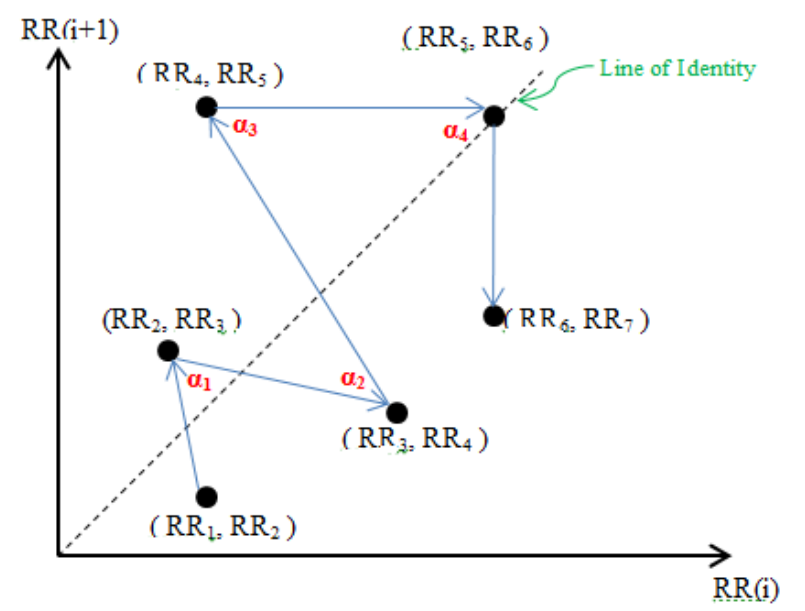

Figure 1. Definition of angle between every three consecutive points in Poincare plot.

\subsubsection{Direction}

The direction of trajectory for every three consecutive points can be one of the following conditions (Figure 2):

1. The points are on straight line.

2. The points have clock-wise orientation.

3. The points have counter clock-wise orientation

Finding one of the specific condition mentioned above is defined as follows:

$$
\operatorname{Dir}_{i}=\left|\begin{array}{ccc}
R R_{i} & R R_{i+1} & 1 \\
R R_{i+1} & R R_{i+2} & 1 \\
R R_{i+2} & R R_{i+3} & 1
\end{array}\right|=\left\{\begin{array}{c}
0 ; \quad \text { on straight line } \\
>0 ; \quad \text { counter clock-wise orientation } \\
<0 ; \quad \text { clock-wise orientation }
\end{array}\right.
$$

In this study, only the sign of Dir is important. So if the points are in counter clock-wise orientation, $\mathrm{Dir}_{\mathrm{i}}$ is coded as +1 and if they are in clock-wise orientation, $\mathrm{Dir}_{\mathrm{i}}$ is coded as -1 .
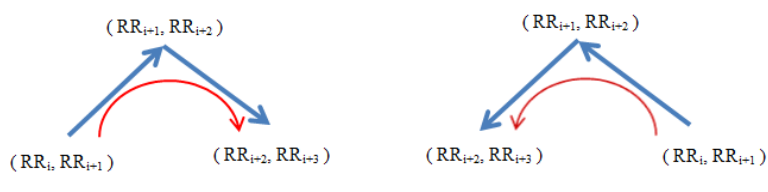

Clock-wise Orientation

Counter Clock-wise Orientation

Figure 2. The direction of trajectory for three consecutive points in Poincare plot.

\subsubsection{Position of Middle Point in Relation to the Line of Identity}

As is shown in Figure 1, the line of identity passes through the origin at an angle of $45^{\circ}$ with horizontal axis [14]. For defining the position of middle point in every three consecutive points in relation to this line, the distance of point to the line $y=x$ is determined as follows:

$$
d_{i}=\frac{R R_{i+1}-R R_{i}}{\sqrt{2}}
$$

If $d_{i}$ is positive, the point is above the line of identity. Negative $d_{i}$ shows that the point is below the line of identity and if $d_{i}$ is zero, the point is on the line of identity. Position of the point was represented by color as third dimension in new ADP map (Figure 3, red, green, and blue were used if middle point was above, on, and below unity line, respectively).

\subsubsection{ADP Map}

ADP map will be used for a better representation of points' temporal behavior in Poincare plot. For creating this map, first the two parameter Angle and Dir were combined by their multiplication. Since Dir is -1 or +1 , the sign of Angle would become positive or negative. We plot this new feature versus $i$ which is the temporal index of points in Poincare plot. The location of the point in relation to the line of identity was added as a third dimension by color. Point above the line of identity, below the line of identity, and on the line of identity were represented by blue, red, and green, respectively.

As it is shown in figure 3 , the first three points in Poincare plot have the angle $163^{\circ}$, and since these points are in counter clock-wise orientation, the parameter Dir is +1 and since the middle point is above the line of identity, it is shown by red color. 

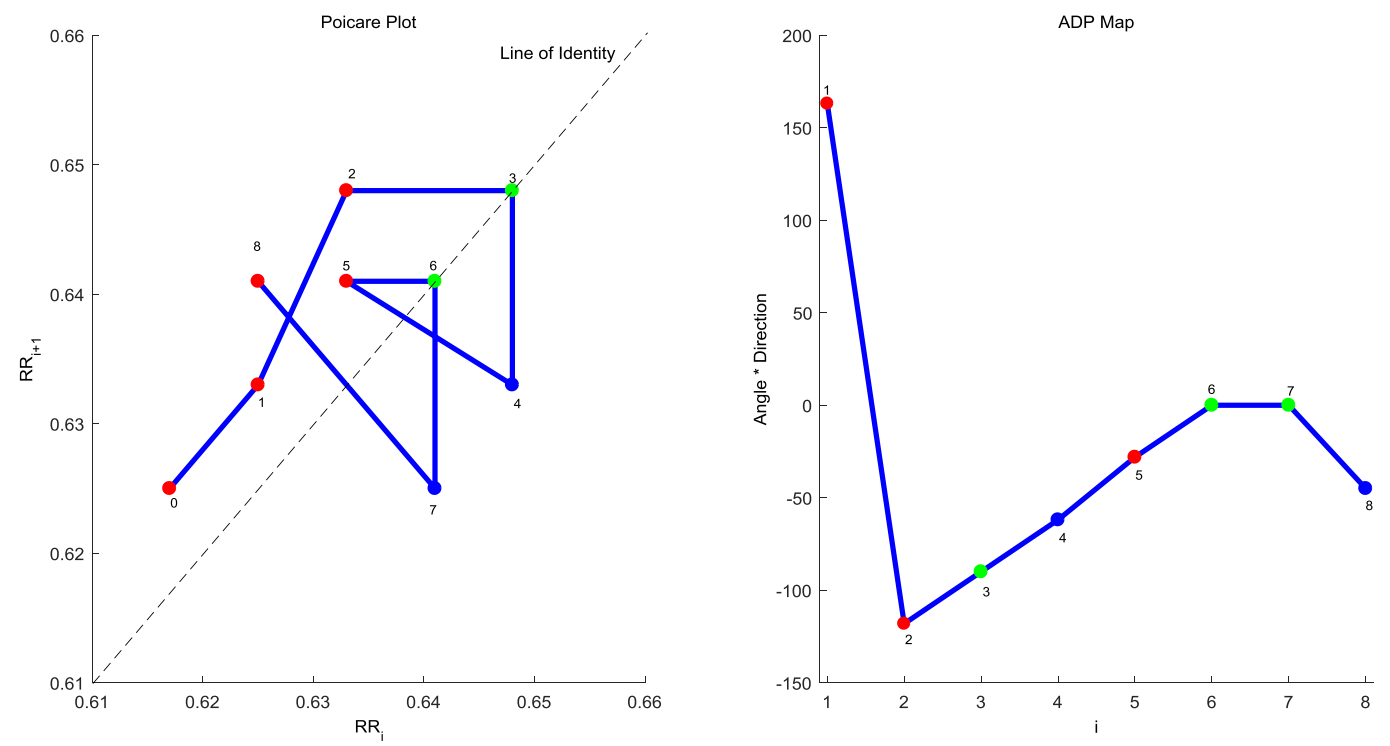

Figure 3. Poincare plot and ADP map of nine consecutive points (Left figure: Poincare plot; Right figure: ADP Map)

\subsection{Statistical Analysis and Classification}

Kruskal-Wallis, a non-parametric version of the classical one-way ANOVA, was used to compare the proposed features between four cardiac conditions. Level of significance was set to 0.05. Extracted ADP features (angle, direction, and position) were used with K- Nearest Neighbor (KNN) classifier for classification of four cardiac condition. KNN was trained on $70 \%$ of data as a train set, and the accuracy was evaluated on $30 \%$ of data as a test set.

\subsection{Data}

In this study, RR intervals for acute myocardial infarction (MI, n=14), Congestive Heart Failure (CHF, $\mathrm{n}=14$ ), Atrial Fibrillation (AF, $\mathrm{n}=14$ ), and Normal Sinus Rhythm (NSR, n=14) were extracted from Physionet for evaluation of the proposed feature set.

The data from Physionet database [15] are used in the experiment. In this study, 14 long-term ECG recordings of subjects in normal sinus rhythm were extracted from Physionet Normal Sinus Rhythm database [15]. Furthermore, NHLBI sponsored Cardiac Arrhythmia Suppression Trial (CAST) RR-Interval Sub-study database for the acute myocardial infarction (MI) data set from Physionet was used for extracting MI data. This database is divided into three different study groups among and the Encainide (e) group data sets was used to extract $14 \mathrm{MI}$ subjects belong to subgroup baseline (no medication) [15] Also, 14 long-term ECG recordings from Physionet Congestive Heart Failure database along with 14 ECG recordings from Physionet Atrial Fibrillation database were used to create $\mathrm{CHF}$ and AF data set, respectively [15]. The original ECG recordings for all groups were digitized at $128 \mathrm{~Hz}$ [15].

\section{Results}

For comparing the results and evaluating the proposed feature set, statistical analysis was used first. Then KNN classifier was used to classify these four groups of arrhythmia by using extracted features. Mean and standard deviation of ADP features are reported in Table 1. The $p$ values from Kruskal-Wallis analysis are shown in Table 2 for ADP features. Angle was significantly different between MI and $\mathrm{AF}$ ( $p$-value $=0.02$ ) as well as $\mathrm{CHF}$ and $\mathrm{AF}$ $(p$-value $<0.05)$. Except $\mathrm{MI}$ and $\mathrm{CHF}$, direction was significantly different in other cardiac group pairs $(p$ value<0.05).

Accuracy, sensitivity, and specificity for KNN classifier for test set is reported in Table 3. Accuracy of classification for four groups was $94.8 \%$ and $95.58 \%$ for training and test set, respectively.

\section{Conclusion}

In this article, a new feature set and map were proposed for capturing temporal information/dynamic of points in Poincare plot. The promising result of this study suggests that new feature set can complement traditional quantifiers of Poincare plot. 
Table 1. Mean and standard deviation of ADP feature set across groups with different cardiac condition (NSR: Normal Sinus Rhythm, MI: Acute Myocardial Infarction, CHF: Congestive Heart Failure, AF: Atrial Fibrillation)

\begin{tabular}{cccc} 
& \multicolumn{3}{c}{ ADP Feature Set } \\
\cline { 2 - 4 } & Angle & Direction & Position \\
\hline NSR & $72.01 \pm 10.71$ & $-0.01 \pm 0.02$ & $-0.18 \mathrm{e}-4 \pm 0.35 \mathrm{e}-4$ \\
\hline CHF & $62.63 \pm 15.38$ & $-0.81 \pm 1.48$ & $-0.04 \mathrm{e}-4 \pm 0.08 \mathrm{e}-4$ \\
\hline MI & $67.10 \pm 13.89$ & $-1.80 \pm 2.97$ & $-0.04 \mathrm{e}-4 \pm 0.65 \mathrm{e}-4$ \\
\hline AF & $78.81 \pm 14.40$ & $-0.01 \pm 0.02$ & $-0.01 \mathrm{e}-4 \pm 0.43 \mathrm{e}-4$ \\
\hline
\end{tabular}

Table 2. P-value for ADP features, significant parameters are highlighted in bold (NSR: Normal Sinus Rhythm, MI: Acute Myocardial Infarction, CHF: Congestive Heart Failure, AF: Atrial Fibrillation).

\begin{tabular}{cccc}
\hline & \multicolumn{3}{c}{$A D P$ Features } \\
\cline { 2 - 4 } Groups & Angle & Direction & Position \\
\hline NSR \& MI & 0.33 & $<\mathbf{0 . 0 5}$ & 0.08 \\
NSR \& CHF & 0.09 & $<\mathbf{0 . 0 5}$ & 0.06 \\
NSR \& AF & 0.19 & $\mathbf{0 . 0 2}$ & 0.06 \\
MI \& CHF & 0.33 & 0.43 & 0.18 \\
MI \& AF & $\mathbf{0 . 0 2}$ & $<\mathbf{0 . 0 5}$ & 0.67 \\
CHF \& AF & $<\mathbf{0 . 0 5}$ & $\mathbf{0 . 0 1}$ & 0.46 \\
\hline
\end{tabular}

Table 3. Classification performance for test set

\begin{tabular}{cccc}
\cline { 2 - 4 } & Accuracy & Sensitivity & Specificity \\
\hline NSR & 94.11 & 100 & 91.66 \\
\hline CHF & 100 & 100 & 100 \\
\hline MI & 88.23 & 75 & 92.30 \\
\hline AF & 100 & 75 & 100 \\
\hline
\end{tabular}

\section{References}

[1] D. McLernon, N. Dabanloo, A. Ayatollahi, V. Majd, and H. Zhang, "A new nonlinear model for generating RR tachograms," Computers in Cardiology, 2004, pp. 481-484, 2004.

[2] N. Dabanloo, D. McLernon, A. Ayatollahi, and V. Majd, "A nonlinear signal processing approach to model heart rate variability," 2004, pp. 64-67.

[3] K. Hnatkova, X. Copie, A. Staunton, and M. Malik, "Numeric processing of Lorenz plots of RR intervals from long-term ECGs*:: Comparison with time-domain measures of heart rate variability for risk stratification after myocardial infarction," Journal of Electrocardiology, vol. 28, pp. 74-80, 1995.

[4] P. Kamen, H. Krum, and A. Tonkin, "Poincare plot of heart rate variability allows quantitative display of parasympathetic nervous activity in humans," Clinical science, vol. 91, pp. 201208, 1996.

[5] T. Thong, "Geometric Measures of Poincare Plots for the Detection of Small Sympathovagal Shifts," in Annual International Conference of the IEEE EMBS, Lyon, France, 2007, vol. 29, pp. 4641-4644: IEEE.

[6] V. Schechtman et al., "Dynamic analysis of cardiac RR intervals in normal infants and in infants who subsequently succumbed to the sudden infant death syndrome," Pediatric research, vol. 31, no. 6, p. 606, 1992.

[7] M. Tulppo, T. Makikallio, T. Takala, T. Seppanen, and H. Huikuri, "Quantitative beat-to-beat analysis of heart rate dynamics during exercise," American Journal of PhysiologyHeart and Circulatory Physiology, vol. 271, no. 1, p. H244, 1996. [8] M. Brennan, M. Palaniswami, and P. Kamen, "Do Existing Measures of Poincare Plot Geometry Reflect Nonlinear Features of Heart Rate Variability?," IEEE Transactions on Biomedical Engineering, vol. 48, no. 11, pp. 1342-1347, 2001.

[9] C. K. Karmakar, A. H. Khandoker, J. Gubbi, and M. Palaniswami, "Complex Correlation Measure: A Novel Descriptor for Poincare Plot," Biomedical Engineering OnLine, vol. 8, pp. 1-12, 2009.

[10] N. Jafarnia Dabanloo, S. Moharreri, S. Parvaneh, and A. M. Nasrabadi, "Application of Novel Mapping for Heart Rate Phase Space and Its Role in Cardiac Arrhythmia Diagnosis," presented at the Computing in Cardiology (CinC 2010), 2010.

[11] S. Moharreri, S. Parvaneh, N. Jafarnia Dabanloo, and A. M. Nasrabadi, "Utilizing Occurrence Sequence of Heart Rate's Phase Space Points in order to Discriminate Heart Arrhythmia," in the 17th Iranian Conference of Biomedical Engineering (ICBME2010), Isfahan, Iran, 2010, vol. 17: IEEE.

[12] S. Moharreri, S. Rezaei, N. Jafarnia Dabanloo, and S. Parvaneh, "Extended Parabolic Phase Space Mapping (EPPSM): Novel Quadratic Function for Representation of Heart Rate Variability Signal," presented at the Computing in Cardiology (CinC 2014), 2014.

[13] N. Jafarnia Dabanloo, S. Moharreri, S. Parvaneh, and A. M. Nasrabadi, "New Representation of Heart Rate and Evaluation of Extracted Geometric Features," presented at the Computing in Cardiology (CinC), Belfast, Northern Ireland, 2010.

[14] C. Karmakar, A. Khandoker, J. Gubbi, and M. Palaniswami, "Defining asymmetry in heart rate variability signals using a Poincaré plot," Physiological measurement, vol. 30, p. 1227, 2009.

[15] A. Goldberger et al., "PhysioBank, PhysioToolkit, and PhysioNet: Components of a new research resource for complex physiologic signals," Circulation, vol. 101, no. 23, p. e215, 2000.

Address for correspondence.

Saman Parvaneh

2 Canal Park, 3rd floor, Cambridge, MA 02141

parvaneh@ieee.org 